\title{
Cardiac Surgery in Jehovah's Witnesses: Another Frontier Overcoming
}

\author{
Alessandro Costetti, Marco Lanfranconi, Marzia Cottini*, Maria Pia Gagliardone, Claudio \\ Francesco Russo \\ Department of Heart and Vessels, Cardiac Surgery Unit and Heart Transplantation Center, Niguarda Hospital, \\ 20162 Milan, Italy
}

*Corresponding Author: Marzia Cottini, Department of Heart and Vessels, Cardiac Surgery Unit and Heart Transplantation Center, Niguarda Hospital, 20162 Milan, Italy, Email:marzia.cottini@ gmail.com

Keywords: cardiac surgery, bloodless surgery, blood conservation, Jehovah's witness.

\section{EDITORIAL}

Cardiac surgery is the most demanding surgery for patients in terms of biological cost. It is not a coincidence that every cardiac surgery unit has an intensive care unit dedicated for the early postoperative monitoring. The most of the cardiac surgery procedures have long operative timing characterized by many sutures in vascular and cardiac tissues and need often the extracorporeal circulation, with inevitable consequences of loss in hematocrit values both in the surgical time and in the following postoperative period [1]. For these reasons, the percentage of blood transfusions in cardiac surgery is still high, easily overcoming the $50 \%$ of the operations. Usually this is not a real problem because of the therapeutic role of blood products in case of postoperative hematological supplement, but it could become an unsurpassable obstacle in those patients that, for many reasons, decided to refuse any kind of blood product transfusion.

According to previous experiences and daily increased social-demands, we decided to start a project in our Cardiac Surgery Department (Niguarda Hospital, Milan, Italy). We started to deal the patients who would like to avoid being transfused for their religious or social guidelines or their convictions or wills but respecting the security of patients and the correctness of surgical procedure.

Firstly, we confronted the most of Jehovah's health exponents to learn exactly what they would accept and what they would reject by their convictions. Secondarily we reviewed the scientific literature and "we were inspired" by non cardiac-surgery articles and experiences that had already dealt about the transfusion problem previously.

Collected scientific literature [1-3] and patients' ideals, we discussed with Anesthesiologists of our Cardiac Anesthesia Department and hence we wrote a protocol to treat the patients that refuse blood products transfusions in the most of cardiac surgery procedures except in some complex operative technique as heart transplantation or aortic arch surgery or debranching surgery in deep hypothermia. We drafted the protocol, that was approved by the hospital and warmly accepted and appreciated by the Jehovah's health exponents, and we started to treat the patients who refused blood products transfusion respecting their wills.

In the protocol we identified three phases in the treatment of the patient:

a. Preoperative Pharmacological Preparation: We prepare the patient by administration of specific supplements to bring them to the intervention with the highest possible hemoglobin value, we intended to reach the cut off from 12,5 to $14 \mathrm{mg} / \mathrm{dl}$.

b. Intraoperative Precautions: We identify the possible and safe hematocrit level for the patient, the blood loss risk. Then we analyze the possibilities of a preoperative bloodletting in the operating room keeping the blood bag connected to the patient or using reduced extracorporeal circulation circuits with centrifugal pump or retropriming and blood recovery techniques or all of them. When it will be possible we 
prefer minimally invasive approaches, like upper "J" ministernotomy or right anterior minithoracotomy to reduce the iatrogenic blood loss.

c. Postoperative Save \& Earn: We use a postoperative blood recovery using a particular drainage circuit connected to the patient in order to recover, wash, treat and reinfuse the blood lost after the intervention. In case of excessive blood loss, we have rather a preventive revision.

After one year we have already treated different types of cardiac pathologies without blood transfusions, we have improved daily our surgical experience and have positive results in reduction of blood transfusion not only in the patients who required no blood transfusion but also in the all elective patients ("bloodless surgery"). The new "Jehovah's Protocol" has been exploited as the daily surgical elective strategies to all the patients with the positive results of reduction of the blood transfusion percentage and with the possibility to face up some clinical conditions with more options than before.

\section{REFERENCES}

[1] Nelson $\mathrm{M}$ et al; Measurement of Blood Loss in Cardiac Surgery: Still Too Much; Ann Thorac Surg. 2018 Mar 3. pii: S00034975(17)31524-2. doi: 10.1016

[2] Olshove $\mathrm{V}$ et al; Comprehensive blood conservation program in a new congenital cardiac surgical program allows bloodless surgery for the Jehovah Witness and a reduction for all patients. Perfusion. 2017 Oct 1:267 65 9117733810. doi:10.1177/0267659117733810.

[3] Tanaka A et al; Cardiovascular surgery in Jehovah's Witness patients: The role of preoperative optimization. J Thorac Cardiovasc Surg. 2015 Oct; 150(4):976-83. e1-3. doi: 10.1016/j.jtcvs.2015.06.059. Epub 2015 Jun 30.

Citation: Alessandro Costetti, Marco Lanfranconi, Marzia Cottini, Maria Pia Gagliardone, Claudio Francesco Russo.Cardiac Surgery in Jehovah's Witnesses: Another Frontier Overcoming. ARC Journal of Cardiology. 2018; 4(1) 1-2. doi: dx.doi.org/11.20431/2455-5991.0401001.

Copyright: (C) 2018Authors. This is an open-access article distributed under the terms of the Creative Commons Attribution License, which permits unrestricted use, distribution, and reproduction in any medium, provided the original author and source are credited. 